\title{
Flow cytometry and compensation of highly autofluorescent cells: the example of mesenchymal stem cells
}

\author{
Anja B. Bohn*, Bjarne K. Moller and Mikkel S. Petersen
}

*Correspondence: anjsch@rm.dk

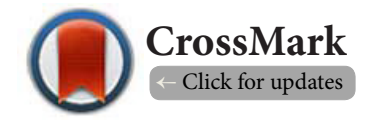

Department of Clinical Immunology, Aarhus University Hospital, Palle Juul-Jensens Boulevard 99, DK-8200, Aarhus, Denmark.

\begin{abstract}
Flow cytometric analysis is a valuable technique for identification and characterization of cells. However, flow cytometric analysis of autofluorescent cells requires careful consideration. In particular, the outcome of fluorescence compensation in the presence of significant autofluorescence poses a challenge to the interpretation of multicolour flow cytometric data. In this paper, we explain the mathematical basis of fluorescence compensation and the effects of autofluorescence, both in theory and applied to mesenchymal stem cells, which are notoriously autofluorescent. We illustrate how failure to understand the consequences of compensation can easily lead to critical errors in data interpretation, particularly when autofluorescence is involved. In the process, the common pitfalls of flow cytometric analysis of mesenchymal stem cells are presented, as are the simple measures necessary to avoid them. Specifically, the counterintuitive concept of "negative fluorescence values" is explained and exemplified, and the phenomenon of "population broadening" is addressed. Researchers must be acutely aware of the effects of compensation on the positioning of cells. We recommend always displaying data in biexponential or logicle transformations and advice to include fluorescence-minus-one controls to establish thresholds of positivity.
\end{abstract}

Keywords: Mesenchymal stromal cells, flow cytometry, cultured cells, cellular structures

\section{Introduction}

Autofluorescence occurs to some extent in all cell types and is part of the intrinsic "background" fluorescence signal in flow cytometry. In some cell types, such as mesenchymal stem cells, autofluorescence is especially high [1]. A high level of background fluorescence complicates flow cytometric analyses, which are often based on multiple, fluorochrome-labelled antibodies and weakly expressed antigens. Indeed, autofluorescence exposes effects of compensation that go unnoticed in "simple" flow cytometry of cells with little autofluorescence (such as lymphocytes) - effects that may catch a flow cytometrist unaccustomed to dealing with autofluorescence unawares.

In the following, we address two common pitfalls in flow cytometric analysis of autofluorescent cells - and describe the simple measures that can be taken to avoid them. As an example of highly autofluorescent cells, we here focus our attention on cultured mesenchymal stem cells, but the principles are universal, and should be considered regardless of cell type.

The most widely used model of spectral overlap compensation does not fully incorporate autofluorescence, which results in counterintuitive data positioning; indeed, highly autofluorescent populations can obtain negative fluorescence values after compensation. In addition, compensation results in broadening of populations, which necessitates the use of fluorescence-minus-one control staining.

\section{Commentary}

The consequence of autofluorescence in compensation Negative fluorescence values after compensation is a fairly common occurrence in flow cytometric analysis of cell types with high levels of autofluorescence (e.g., mesenchymal stem cells). At first glance, the concept of negative fluorescence is counterintuitive, and researchers may instinctively begin to doubt the validity of the compensation, the flow cytometer, or suspect a "bug" in the flow cytometric software. Worse, the researcher may be sufficiently perplexed to conclude that flow cytometry of such cells "doesn't work" and abandon flow cytometric analyses altogether. However, negative fluorescence values do make sense when one considers what compensation does. 
The classical approach to compensation involves recording the signals of single-stained compensation controls to determine the degree of spectral overlap between fluorochromes and detectors. Compensation is typically done by adjusting the values of the spectral overlap matrix elements (algorithmically by iteration or in rare cases manually by "visual inspection"), until the compensated median fluorescence intensity (MFI) of labelled cells (or beads) matches the compensated MFI of unlabelled cells in all detectors except the one assigned to the fluorochrome of the particular single-stained population.

Although mathematical models that incorporate autofluorescence exist [2], flow cytometry software algorithms for compensation typically rely on a simpler model, in which the signal on detector $j$ can be described as:

$$
S_{j}=\sum_{i=1}^{n} M_{i j} \times F_{i}
$$

where $n$ is the number of fluorochromes/detectors, $M_{i j}$ is the spectral overlap matrix element representing the relative signal of the $i$-th fluorochrome in the $j$-th detector, and $F_{i}$ represents the amount of the $i$-th fluorochrome. The equivalent equation in matrix notation is

$$
S=M F
$$

where $S$ is the column vector of signals detected, $M$ is the spectral overlap matrix, and $F$ is a vector representing the amounts of fluorochromes. Correction for spectral overlap then involves finding the inverse of matrix $M$ (i.e., the compensation matrix $M^{-1}$ ), and applying it to the signal vector to obtain the F-vector (which is used for data visualization), that is:

$$
S=M F \Leftrightarrow M^{-1} S=M^{-1} M F \Leftrightarrow M^{-1} S=F
$$

This operation is essentially a linear transformation affecting the scale and relative angles of the axes of the coordinate system while leaving the origin unaffected. As such, classical compensation does not include translation of the coordinate system. This fact can lead to surprises in data visualization when autofluorescence is involved, as described in the following.

Let us consider a hypothetical staining experiment involving only two fluorochromes $\left(F_{1}\right.$ and $\left.F_{2}\right)$ and two detectors $\left(S_{1}\right.$ and $S_{2}$ ). For the sake of simplicity, let us further assume that the spectral overlap from fluorochrome $F_{1}$ into $S_{2}$ is $100 \%$ (that is, the fluorescence emission from $F_{1}$ is detected equally well by both detectors) whereas the spectral overlap from $F_{2}$ into $S_{1}$ is $0 \%$ (that is, $F_{2}$ emissions can only be detected by the $S_{2}$ detector). However, there is a considerable autofluorescence component present in the $S$, detector, and no autofluorescence in the $S_{2}$ detector. Let $A_{1}$ denote the MFI of the autofluorescent cells in the $S$, detector in an unstained sample.

Thus, assume that an unstained cell has a signal-vector of $\left[S_{1}, S_{2}\right]=[50,0]$, so the entire signal actually represents autofluorescence (i.e., $A_{1}=50$ ). Now imagine that the same cell single-stained with fluorochrome $F_{1}$ produces a signal-vector of $\left[S_{1}, S_{2}\right]=[100,50]$. Here, the $S_{1}$-component is thus the sum of an autofluorescence of 50 and an actual fluorescence emission from the $F$, fluorochrome corresponding to a value of 50; and the $S_{2}$-component consists entirely of spectral overlap from fluorochrome $F_{1}$, which in this case is $100 \%$ of 50 . This situation is illustrated in Figure $\mathbf{1 A}$, where the light blue dot represents the unstained cell and the dark blue dot represents a cell stained only with fluorochrome $F_{1}$.

The $\mathrm{X}$-axis is the signal on fluorescence detector 1 , and the $\mathrm{Y}$-axis is the signal on fluorescence detector 2 . The slope of the red line is - by definition - the spectral overlap from fluorochrome 1 into detector 2 , which in this case has a value of $1(100 \%)$. Notice that the cells in this example are autofluorescent in detector 1 only. To the right in Figure $1 \mathrm{~A}$ the "basic model" of spectral overlap is illustrated: The vector to the left of the equal sign represents the "raw" detector values; the vector to the right represents the compensated values; and the matrix represents the spectral overlap matrix. Within the spectral overlap matrix, each fluorochrome is represented as a single column, and each row represents the relative signal of the fluorochrome in each detector. Here, the spectral overlap matrix is set to "uncompensated" values (i.e., compensated data equal uncompensated data).

The classical approach to compensation of the spectral overlap from fluorochrome $F_{1}$ into the $S_{2}$ detector is essentially a change of basis of the coordinate system, so that the new $\mathrm{X}$-axis basis vector points is parallel to the vector formed by connecting the positions of the unstained and $F_{1}$-only-stained samples. When data points are expressed in terms of the new coordinate basis vectors, the contribution of "irrelevant" fluorochromes is eliminated.

Geometrically, this transformation can be thought of as vertical shearing of data; i.e., each data point is displaced on the $\mathrm{Y}$-axis by an amount proportional to the position on the $\mathrm{X}$-axis, to a degree that exactly counteracts the effect of spectral overlap.

The procedure is illustrated in Figure 1B (incomplete compensation) and $1 \mathrm{C}$ (proper compensation).

To the left in Figure 1B an insufficient compensation basis is illustrated graphically using the data points of Figure $\mathbf{1 A}$. Notice that the slope of the F1-axis is only half the slope of the red line. The basic model of spectral overlap, with the spectral overlap matrix used in the plot on the left, is illustrated to the right (top) where the new $X$-axis basis is the vector $(1,0.5)$, and the $\mathrm{Y}$-axis basis vector is unchanged $(0,1)$. To transform a set of coordinates from one basis to another, one must right-multiply with the inverse of the matrix composed of the new basis vectors expressed in the old coordinates. This inverse matrix of the spectral overlap matrix is called the "compensation matrix" and is illustrated to the right (middle).

The effect of applying the compensation matrix to singlestained cells and unstained cells is also illustrated to the right (bottom). Notice the different compensated $\mathrm{Y}$-axis values of stained (dark blue arrow) and unstained (light blue arrow) populations. 


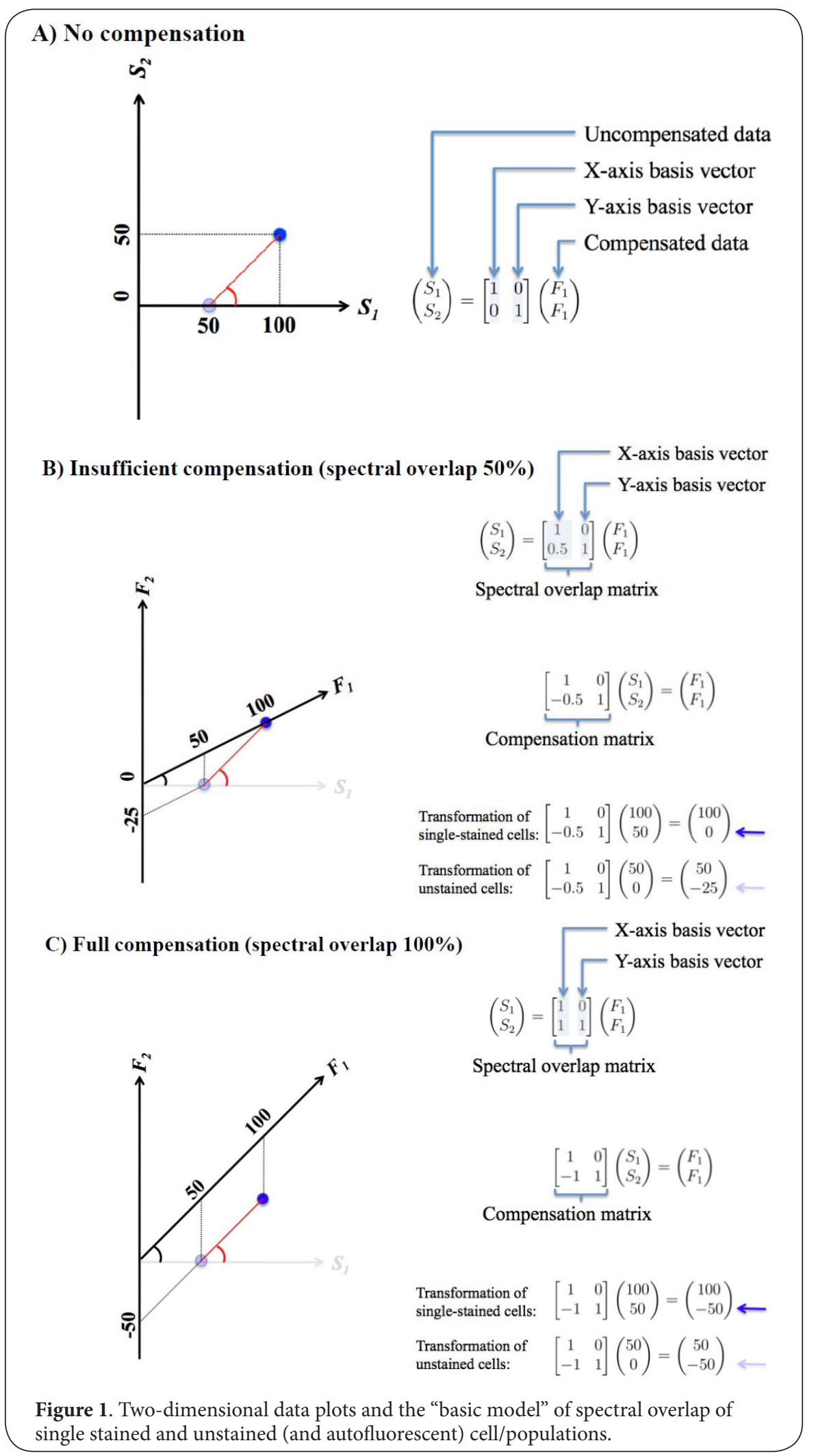

The figure shows an "undercompensated" situation, in which

tor is erroneously assumed to be $50 \%$. In this case, the new the spectral overlap from fluorochrome $F_{1}$ into the $S_{2}$ detec$\mathrm{X}$-axis basis vector is $(1,0.5)$. This situation can easily arise 
when compensation is based on a comparison between single-stained cells that are autofluorescent and unstained cells that are not (i.e., dissimilar levels of autofluorescence in positive and negative populations).

As can be seen, the position of the single-stained cell in the new, compensated coordinate system is $(100,0)$, whereas the position of the unstained cell is $(50,-25)$. Notice how the transformation has reduced the $Y$-value of the single stained cells by 50 to a value of 0 , but also reduced the $Y$-value of the unstained cells by 25 to a value of -25 . In other words, the $Y$ values of stained and unstained cells are still not identical after compensation, so the compensation is insufficient.

This is a major pitfall in flow cytometry in general, and in mesenchymal stem cell analysis in particular. The remedy, fortunately, is simple: for proper compensation, stained and unstained cells/beads must have equal levels of autofluorescence.

It is perfectly possible to perform good compensation by staining cells with a high level of autofluorescence, as long as those same cells can still be identified in an unstained state. One should not, however, be tempted to base compensation on a population of positive, highly autofluorescent cells and another population of negative cells with low autofluorescence. The simplest way to isolate the contribution of the fluorochrome from the contribution of the autofluorescence is-obviously-to have the same baseline of autofluorescence in both positive and negative samples.

The use of compensation beads is generally recommended to ensure similar levels of autofluorescence, and comes with the extra benefits of a strong, positive signal, and a small staining variation within the positive population. But it is certainly possible to obtain a proper spectral overlap matrix with some fluorochromes on beads and others on cells, as long as the proper negative population is used in each case.

A proper compensation of our example is illustrated in Figure 1C. Notice to the left in the figure that the slope of the F1-axis now matches the slope of the red line. The basic model of spectral overlap with the spectral overlap matrix used in this plot is shown to the right (top) where the new $X$-axis basis is the vector $(1,1)$, and the $Y$-axis basis vector $(0,1)$ is unaffected by the transformation. The Compensation matrix, found by inversion of the spectral overlap matrix is shown in the middle to the right and the effect of applying the compensation matrix to single-stained cells and unstained cells is illustrated in the bottom (right). Notice the identical compensated $\mathrm{Y}$-axis values of stained (dark blue arrow) and unstained (light blue arrow) populations.

In our particular example, the shear angle is 45 degrees (corresponding to an overlap/slope/spillover-coefficient $M_{12}$ of 1). In general, the angle is obtained simply as $\arctan (M)$ where $M$ is the relevant element of the spectral overlap matrix.

Notice how this vertical shearing transformation has the intended effect of rendering the compensated $Y$-axis values of $F$-single-stained cells equal to the compensated $Y$-axis values of unstained cells-so rendering the compensated $Y$ axis independent of $F_{1}$ staining.

But the compensation also has the somewhat undesirable side-effect of introducing negative "fluorescence"-values: the unstained cell had an $\mathrm{Y}$-axis of 0 before the compensation, but ends with an $Y$-axis value of -50, as does the $F_{1}$-singlestained cell.

This raises another important point when compensating autofluorescent cells: if these compensated data are represented on a simple log scale, negative events will be invisible (or piled up in the lowest channel, depending on the software). In an extreme situation, this could lead to misinterpretation of data. The simple remedy is to always use biexponential or logicle [3] representation of compensated data to ensure that all data are visible, even when values are highly negative.

It is important to emphasise that negative values after compensation per se do not imply over-compensation, as is sometimes erroneously surmised. Rather, they arise as a natural consequence of the mathematical transformation. Differences in compensated values are still proportional to differences in the amount of fluorochrome bound to the cell-but the absolute value is, of course, largely arbitrary, and depends (among other factors) on the level of autofluorescence in the cell.

To avoid excessively negative values, one could theoretically move the coordinate system origin to the centre of the unstained cell population before applying the compensation matrix (or adjust voltage/gain settings to fix the centre of the unstained, autofluorescent cells at the coordinate system origin before recording compensation controls - never after recording, since adjusting voltage/gain will change the compensation needed.). This would leave the position of the unstained cells unaffected by compensation. In actual practice, however, flow cytometry acquisition software rarely permits such data translation, or is fixed in a log-display mode.

An entirely different approach is to devote a fluorescence detector to measure autofluorescence directly on a cell-bycell basis, and "compensate" away the autofluorescence in other channels [4]. Although occasionally useful, the method requires careful selection of the "autofluorescence" detector and may severely limit the number and type of fluorochromes available for staining.

Compensation of autofluorescent mesenchymal stem cells To illustrate the points raised, consider the following data from staining of fourth-passage mesenchymal stem cells (MSC).

Figure 2A shows data of unstained (left) and single-stained (middle) MSC that have been stained with an anti-CD90 antibody conjugated with a PerCP-Cy5.5 fluorochrome (events have been gated on FSC/SSC to exclude debris and non-MSC remnants). On the particular flow cytometer used, the best detector available for PerCP-Cy5.5 emissions is the PerCP detector (X-axis), but a strong spectral overlap is evident in the PE-Cy7 detector (Y-axis) as illustrated in the middle and 
Bohn et al. Stem Cell Biology and Research 2015,

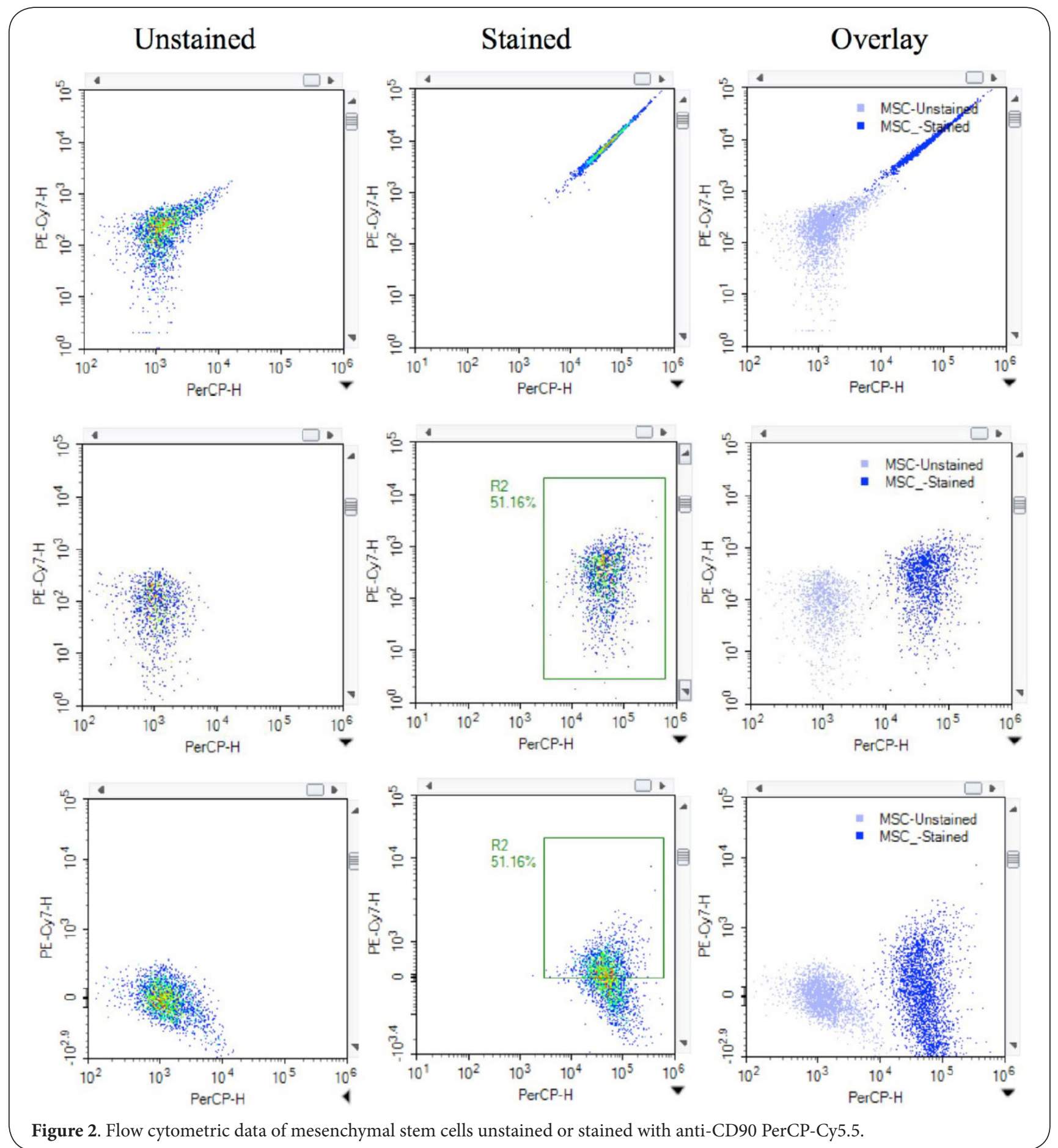

right part of Figure 2A. Furthermore, MSC have strong autofluorescence in the PerCP-channel, but only moderate/low autofluorescence in the PE-Cy7 channel. Such combinations set the stage for "negative fluorescence": we need to correct for a major spectral overlap from a channel with high autofluorescence into a channel with relatively low autofluorescence.

And so, after applying a compensation matrix (typically obtained from staining of compensation beads), we arrive at the situation depicted in Figure 2B.

At first glance, all is in reasonably good order - although one might be tempted to conclude that the spillover coefficient has been slightly underestimated (it is approximately $16 \%$ ), inasmuch as the stained population still seems to have a slightly higher median Y-axis position than the unstained cells - but 
surely nothing terribly amiss. A careless flow cytometrist might even make slight, manual adjustments to the compensation matrix to "set matters straight" based on these plots.

But a closer look reveals that something is very wrong. When a gate is placed around the visible population of cells after compensation, only slightly more than $50 \%$ of the total stained cells are included. The reason is simple: compensation has introduced negative fluorescence values on the Y-axis, and such values cannot be displayed on a logarithmic scale, leading to "loss" of roughly half the events. Fortunately, switching to biexponential scaling saves the day (illustrated in Figure 2C).

The biexponential scale reveals that the compensation is indeed correct: the median Y-axis value of single-stained MSC after compensation is equal to the median $Y$-axis value of unstained MSC after compensation (that value is -32 , incidentally).

The biexponential axis also exposes another consequence of compensation-population broadening-which is discussed in the following.

\section{The importance of fluorescence-minus-one controls} In a perfect flow cytometer, there would be no error in fluorescence measurements, and one could safely assume that the measurements were "true" indications of the actual fluorescences on each cell. The "width" (or spread, or variation) of a population of cells on any given parameter would then reflect the actual, physical variation of that parameter within the population, and nothing else. In this case, compensation would not affect the "width" or shape of the population (other than the purely visual changes introduced by redefining the basis of the coordinate system by shearing transformation) and the "width" of the stained cell population would closely resemble that of the unstained cells, both before and after compensation (insofar as a "perfect" compensation truly eliminates the contribution of irrelevant fluorochromes on each individual cell, leaving only the natural variation of the background/autofluorescence).

In reality, measurements are always less-than-perfect, and there is some uncertainty associated with each fluorescence measurement - at the very least, there is always the intrinsic "photon count"/Poisson uncertainty. This is true of the measurements used to determine spectral overlap (so the compensation matrix itself is not "perfect"), and of the measurements of each cell on each parameter.

Imagine, for example, that the uncertainty associated with the actual fluorescence measurements of each cell is on the order of one "pixel" in the plot (that is, each dot in the plot could be "off" by plus or minus one pixel). Because of the logarithmic nature of the axes, this "one-pixel error" could easily mean an absolute uncertainty of several thousand (arbitrary) fluorescence units in brightly stained populations (i.e., populations far from the origin). When such populations are "brought down" towards background levels by compensation, the transformation does not reduce the uncertainty of the original measurements. Indeed, an uncertainty of one pixel in the uncompensated sample is "magnified" by displaying the population further down on a logarithmic (or biexponential) scale, where a given absolute range takes up more and more space.

As a result, populations are "widened" by compensation. It is important to understand that this effect does not reflect a deficiency of compensation as such. Rather, it is the unavoidable consequence of the fact that each compensated event relies on several measurements: (at least) two measurements of fluorescence, each with its own, independent error; in addition to a systematic error that may or may not arise from a slightly incorrect compensation matrix. These errors are additive, and nothing can be done about it (short of constructing ever more "perfect" fluorescence detectors).

The consequences of population broadening are clearly visible in our example with mesenchymal stem cells in Figure $\mathbf{2 C}$. Notice (in the overlay-plot) how the compensated, singlestained population extends both above and below the more "compact" population formed by the unstained population, even though compensation is "perfect" (in the sense that the median fluorescences of the $Y$-axis are equal).

Importantly, the unstained sample does not tell us at what level we should consider a cell "positive" on the Y-axis after compensation.

Looking at the unstained cells of Figure $2 \mathrm{C}$, we might be tempted to surmise that anything with a PE-Cy7 signal above, say 500 fluorescence units, should be considered positive. After all, all unstained cells lie below 500 , so the claim does not seem unreasonable at first glance. However, if we were to apply this threshold to the single-stained population, a sizable fraction of the cells would be PE-Cy7 positive, simply because of population broadening.

The answer is to base such thresholds on so-called "fluorescenceminus-one" (FMO) controls [5]. These controls are simply cells stained with all antibodies except one-so in a sense, they are "reverse" compensation controls (i.e., omitting one antibody at a time, rather than including one antibody at a time). If we want to determine the threshold at which a cell should be considered truly PE-Cy7 positive, we would stain the cells with all antibodies except PE-Cy7, apply the compensation matrix (and thus introduce the population broadening arising from the combined spectral overlap of all fluorochromes), and then consider the position of these compensated cells on the PE-Cy7 axis. This approach not only addresses autofluorescence, but also the added uncertainties that arise from compensation of all other fluorochromes in the sample.

In a two-colour experiment such as that presented, the single-stained control also conveniently serves as an FMO control-but more complicated situations obviously require separate single-stained compensation controls and FMO controls.

What FMOs do not accomplish, however, is to settle doubts of unspecific antibody binding. For this purpose, isotype controls are sometimes useful. The chief limitation of isotype 
Bohn et al. Stem Cell Biology and Research 2015,

controls is the difficulty of finding an isotype control antibody preparation that is truly comparable to the "real" antibody in terms of antibody concentration, isotype, conjugation efficiency, and fluorochrome composition.

\section{Conclusion}

Autofluorescent cells, including mesenchymal stem cells, are indeed amenable to flow cytometric analyses, in spite of high levels of autofluorescence. However, researchers must be acutely aware of the effects of compensation on the positioning of cells (and thus always display data in biexponential or logicle transformations). Compensation controls can be based on beads (recommended) or cells, or a combination thereof, with no theoretical implications for the compensation matrix, insofar as the matrix is solely based on slopes. Compensations must, however, compare stained and unstained populations with identical autofluorescences. Finally, researchers are advised to include fluorescence-minus-one controls to establish thresholds of positivity.

\section{Competing interests}

The authors declare that they have no competing interests.

Authors' contributions

\begin{tabular}{|l|c|c|c|}
\hline Authors' contributions & ABB & BKM & MSP \\
\hline Research concept and design & $\checkmark$ & $\checkmark$ & $\checkmark$ \\
\hline Collection and/or assembly of data & $\checkmark$ & -- & -- \\
\hline Data analysis and interpretation & $\checkmark$ & -- & $\checkmark$ \\
\hline Writing the article & $\checkmark$ & -- & $\checkmark$ \\
\hline Critical revision of the article & -- & $\checkmark$ & $\checkmark$ \\
\hline Final approval of article & -- & -- & $\checkmark$ \\
\hline Statistical analysis & -- & -- & -- \\
\hline
\end{tabular}

\section{Acknowledgement and funding}

Financial support for this study was provided

by Karen Elise Jensens Foundation.

Publication history

EIC: Prasad S. Koka, Haffkine Institute for Training, Research \& Testing, India.

Received: 21-Oct-2015 Final Revised: 23-Nov-2015

Accepted: 30-Nov-2015 Published: 11-Dec-2015

\section{References}

1. Anwer AG, Gosnell ME, Perinchery SM, Inglis DW and Goldys EM. Visible $532 \mathrm{~nm}$ laser irradiation of human adipose tissue-derived stem cells: effect on proliferation rates, mitochondria membrane potential and autofluorescence. Lasers Surg Med. 2012; 44:769-78. | Article | PubMed

2. Bagwell $C B$ and Adams $E G$. Fluorescence spectral overlap compensation for any number of flow cytometry parameters. Ann N Y Acad Sci. 1993; 677:167-84. | Article | PubMed

3. Tung JW, Parks DR, Moore WA and Herzenberg LA. New approaches to fluorescence compensation and visualization of FACS data. Clin Immunol. 2004; 110:277-83. | Article | PubMed

4. Roederer M and Murphy RF. Cell-by-cell autofluorescence correction for low signal-to-noise systems: application to epidermal growth factor endocytosis by 3 T3 fibroblasts. Cytometry. 1986; 7:558-65. | Article | PubMed

5. Roederer M. Spectral compensation for flow cytometry: visualization artifacts, limitations, and caveats. Cytometry. 2001; 45:194-205. | Pdf | PubMed

\section{Citation:}

Bohn AB, Moller BK and Petersen MS. Flow cytometry and compensation of highly autofluorescent cells: the example of mesenchymal stem cells. Stem Cell Biol Res. 2015; 2:4. http://dx.doi.org/10.7243/2054-717X-2-4 